\title{
Cerebral Adrenoleukodystrophy
}

National Cancer Institute

\section{Source}

National Cancer Institute. Cerebral Adrenoleukodystrophy. NCI Thesaurus. Code C142852.

A subtype of adrenoleukodystrophy (ALD) occurring in approximately 40 percent of boys with ALD, primarily affecting the cerebrum, resulting in rapidly declining neurocog nitive function and in most patients, premature death. 